\title{
Clinicaenton Practice
}

Activity index, Henoch-Schönlein nephritis $\mathrm{c} 72$

Acute care setting, glomerular filtration rate estimation $\mathrm{c} 1$

- renal failure c211

Adiponectin c18

Albuminuria c116

Aluminum serum levels, hemodialysis patients c122

Angiography, vasoactive factor urinary excretion c150

Angiotensin-converting enzyme inhibitors c139

Arteriovenous fistula c39

- $\quad$, risk factors c39

Atherosclerosis c18, c109, c134, c180

Bone alkaline phosphatase c122

Calcitriol c94

Calcium channel blockers c139

Cardiovascular disease c47, c116

Carnitine c174

Carotid artery atherosclerosis c180

- intima-media thickness c134

Cephalosporins c58

Chronic renal disease c47, c79

- - failure c53, c139, c207

Citrate anticoagulation c211

Collagen type $1 \mathrm{C}$-terminal telopeptide c9

Continuous veno-venous hemodialysis c211

Contrast media, vasoactive factor urinary excretion c150

Coronary flow reserve c200

C-reactive protein $\mathrm{c} 18, \mathrm{c} 190$

Creatinine clearance c128, c190

Cyclophosphamide c25

Cyclosporine A c65

Cytokines c100

Delayed graft function c65

Diabetes c1, c116

- complications c116

Dihydrorhodamine c100

Donor renal function measurement c128

Drug side effects c139

Early arteriovenous fistula failure c39

Elderly hospitalized patients, glomerular filtration rate estimation $\mathrm{c} 1$
Endothelin-1 c150

End-stage renal disease c18, c100

Erythropoietin, recombinant human c79, c87

Extended-spectrum $\beta$-lactamase c58

Fabry disease c33

Fibroblast growth factor-23 c94

Flow cytometry, intracellular oxidative stress c100

Formula estimation, glomerular filtration rate $\mathrm{c} 1$

Fractional shortening, c-reactive protein c190

$\alpha$-Galactosidase A c33

Glomerular filtration rate c1, c128

Hematocrit c190

Hemodialysis c9, c18, c33, c53, c58, c79, c87, c94, c100, c109, c122, c155, c174, c180, c200, c207, c211

-, Israel c58

-, survival c18

Henoch-Schönlein nephritis c72

Hepatitits $\mathrm{C}$ virus infection c207

High-density lipoprotein subclasses c174

High-resolution B-mode ultrasonography, carotid intima-media thickness $\mathrm{c} 134$

Homocysteine c180

Hyperparathyroidism c53, c94

Hypertension c139

Hypo-/hypercalcemia c211

Hyponatremia c211

IgA/C3 ratio c72

Immunodeficiency, end-stage renal disease c100

Innate antiviral immunity c207

Intact parathyroid hormone $\mathrm{c} 122$

Intima-media thickness c18, c109, c134

Ionized calcium c9

Iron $\mathrm{c} 155$

Ischemia, non-occlusive, mesenteric c87

$\beta$-Lactamase c58

Left ventricular mass index c190

Lipoprotein, oxidized, low-density c109

Lymphocytes, oxidative stress $\mathrm{c} 100$

Metabolic alkalosis c211

Mortality, pre-dialysis rHuEPO impact c79
Neopterin c134

Nephrology services, access c168

Nephrotic syndrome c25

Nitric oxide c150

Oscillation, parathyroid hormone secretion c9

Osteocalcin c9

Oxidative stress $\mathrm{c} 100$

Parathyroidectomy c53

Parathyroid hormone c9, c94, c122

Percutaneous ethanol injection, uremic hyperparathyroidism c53

Phosphate, restless legs syndrome c155

Plaque score, atherosclerosis c109

Pre-dialysis patients, C-reactive protein c190

Prediction equations, glomerular filtration rate $\mathrm{c} 128$

Prevalence, restless legs syndrome c155

Proteinuria c139

Pulse wave velocity, atherosclerosis c109

Rabbit anti-human thymocyte globulin c65

Recombinant human erythropoietin c79, c87

Reference glomerular filtration rate, donor renal function measurement $\mathrm{c} 128$

Referral rates, out-reach clinics c168

Renal failure c33, c211

- osteodystrophy c122

- transplant c65

Restless legs syndrome, hemodialysis patients $\mathrm{c} 155$

Risk factors, restless legs syndrome c155

Screening, Fabry disease c33

Secondary hyperparathyroidism c94

Serologic marker, Henoch-Schönlein nephritis $\mathrm{c} 72$

Serum albumin, pre-dialysis patients c190

Sodium excretion, human contrast media exposure $\mathrm{c} 150$

Superoxide dismutase, extracellular c109

Vascular medial calcification c87 